\title{
ASYMPTOTIC DISTRIBUTION OF EIGENVALUES OF BLOCK TOEPLITZ MATRICES
}

\author{
M. ROSENBLATT ${ }^{1}$
}

Communicated by Edwin Hewitt, June 1, 1960

Let $g(\lambda),-\pi \leqq \lambda \leqq \pi$, be a $p \times p(p=1,2, \cdots)$ matrix-valued Hermitian function. Further $g(\lambda)$ is bounded on $[-\pi, \pi]$, that is, its elements are bounded on $[-\pi, \pi]$. The Fourier coefficients

$$
a_{k}=\frac{1}{2 \pi} \int_{-\pi}^{\pi} e^{i k \lambda} g(\lambda) d \lambda, \quad k=0, \pm 1, \cdots,
$$

are then bounded in $k$. We call the $n p \times n p$ matrix

$$
A_{n}=\left(a_{j-k} ; j, k=1, \cdots, n\right)
$$

(an $n \times n$ matrix of the $p \times p$ blocks $a_{j-k}$ ) the $n$th section block Toeplitz matrix generated by $g(\lambda)$. Notice that the block Toeplitz matrix $A_{n}$ is generally not Toeplitz. Our interest is in obtaining the asymptotic distribution of eigenvalues of $A_{n}$ as $n \rightarrow \infty$. The proof is suggested by an argument given in the one-dimensional case $(p=1)$ (see [3]) and is based on results in the multidimensional prediction problem [5].

If the real number $\alpha$ is sufficiently small in absolute value $f(\lambda)$ $=\left[I_{p}+\alpha g(\lambda)\right]$ is positive definite for all $\lambda$ and bounded $\left(I_{p}\right.$ is the identity matrix of order $p$ ). Let $R_{n}=I_{n p}+\alpha A_{n}$ be the $n$th section block Toeplitz matrix generated by $f(\lambda)$. Further denote the $(i, j)$ th block element ( $p \times p$ matrix), $i, j=1, \cdots, n$, of the inverse $R_{n}^{-1}$ of $R_{n}$ by ${ }_{n} r_{i, j}^{(-1)}$. The basic result on the determinant of the prediction error covariance matrix in the multidimensional prediction problem [5] tells us that

$$
\lim _{n \rightarrow \infty} \operatorname{det}\left({ }_{n} r_{11}^{(-1)}\right)^{-1}=(2 \pi)^{p} \exp \left\{\frac{1}{2 \pi} \int_{-\pi}^{\pi} \log \operatorname{det}\left(\frac{f(\lambda)}{2 \pi}\right) d \lambda\right\}
$$

since $f(\lambda) / 2 \pi$ can be regarded as the spectral density function of a $p$-vector weakly stationary stochastic process. However,

$$
\operatorname{det}\left({ }_{n} r_{11}^{(-1)}\right)^{-1}=\operatorname{det}\left(R_{n}\right) / \operatorname{det}\left(R_{n-1}\right)=\sigma_{n}^{2}
$$

(see $\left[1\right.$, p. 21]). Let $\lambda_{\nu, n}, \nu=1, \cdots, n p$, be the eigenvalues of $A_{n}$.

1 This research was supported in part by the U. S. Army Signal Corps. 
Now

and

$$
\operatorname{det}\left(R_{n}\right)=\prod_{\nu=1}^{n p}\left(1+\alpha \lambda_{\nu, n}\right)
$$

Thus

$$
\lim \log \sigma_{n}^{2}=\lim \frac{1}{n} \sum_{k=1}^{n} \log \sigma_{k}^{2}=\lim \frac{1}{n} \sum_{\nu=1}^{n p} \log \left(1+\alpha \lambda_{\nu, n}\right) .
$$

$$
\lim _{n \rightarrow \infty} \frac{1}{n} \sum_{\nu=1}^{n p} \log \left(1+\alpha \lambda_{\nu, n}\right)=\frac{1}{2 \pi} \int_{-\pi}^{\pi} \log \operatorname{det}\left(I_{p}+\alpha g(\lambda)\right) d \lambda .
$$

On taking $s_{n, k}=\sum_{\nu=1}^{n p} \lambda_{\nu, n}^{k}$, it is readily seen that

$$
\lim \sum_{k=1}^{\infty} \frac{(-1)^{k-1}}{k} \frac{s_{n, k}}{p n} \alpha^{k}=\sum_{k=1}^{\infty} \frac{(-1)^{k-1}}{k} \frac{1}{2 \pi p} \int_{-\pi}^{\pi} \operatorname{tr}\left(g^{k}(\lambda)\right) \alpha^{k} d \lambda
$$

for $|\alpha|$ sufficiently small $(\operatorname{tr}(A)$ denotes the trace of $A$ ). This cannot hold unless

$$
\lim _{n \rightarrow \infty} \frac{s_{n, k}}{p n}=\frac{1}{2 \pi p} \int_{-\pi}^{\pi} \operatorname{tr}\left(g^{k}(\lambda)\right) d \lambda .
$$

Let $\mu_{1}(\lambda), \cdots, \mu_{p}(\lambda)$ be the eigenvalues of $g$ at $\lambda$, let us say for convenience in order of magnitude. Then relation (2) implies that the asymptotic distribution of eigenvalues $\lambda_{\nu, n}=1, \cdots, p n$, is given by

$$
\lim _{n \rightarrow \infty} \frac{\text { number of eigenvalues } \leqq a}{p n}=\frac{1}{p} \sum_{j=1}^{p} P\left[\mu_{j}(X) \leqq a\right]
$$

where $X$ is a random variable uniformly distributed on $[-\pi, \pi]$. Aside from the interest in this result for its own sake, it is clear that it suggests a number of good approximations for the joint distribution of spectral estimates in the case of multidimensional normal stationary processes $[2 ; 4]$.

\section{REFERENCES}

1. F. R. Gantmacher, The theory of matrices, vol. 1, New York, Chelsea Publishing Co., 1959.

2. N. R. Goodman, On the joint estimation of the spectra, cospectrum and quadrature spectrum of a two-dimensional Gaussian process, Scientific Paper No. 10, Engineering Statistics Laboratory, New York University, 1957, p. 168.

3. U. Grenander and G. Szegö, Toeplitz forms and their applications, University of California Press, 1958.

4. M. Rosenblatt, Statistical analysis of stochastic processes with stationary residuals, The H. Cramér Volume, Stockholm, Almquist and Wiksell, 1959.

5. N. Wiener and P. Masani, The prediction theory of multivariate stochastic processes, Acta Math. vol. 98 (1957) pp. 111-150.

BROWN UNIVERSITY 